\title{
Assessment of the Effects of a Community Pharmacy Women's Health Education Program on Management of Menopause Survey Scores
}

\author{
MARIO M. ZEOLLA, PharmD, BCPS, and JENNIFER CERULLI, PharmD, BCPS
}

\begin{abstract}
OBJECTIVE: This study examined the effect of a community pharmacy-based menopause education program on scores of the Management of Menopause (MoM) survey. The MoM survey is a tool administered to managed care organization members by the National Committee for Quality Assurance to determine the level of menopause-related education offered by their health care providers. The primary outcome was comparison of the median MoM survey scores of participants at baseline, 3 months, and 1 year posteducation.

METHODS: Women aged 47 to 55 years who were able to provide informed consent were enrolled. Subjects completed a baseline MoM survey. Trained pharmacists working in 7 pharmacies conducted one-on-one education sessions regarding the consequences of menopause, treatment options, and the known risks and benefits of each option. Follow-up MoM surveys were administered by mail at 3 months and 1 year posteducation. The survey is scored on a 100-point scale for an overall composite score and includes 3 subsections: exposure, breadth, and personalization of counseling.

RESULTS: A total of 31 subjects were enrolled, with 24 and 16 completing both baseline and 3-month or 1-year MoM follow-up surveys, respectively. Median 3-month composite MoM survey scores $(86.1 ; 95 \% \mathrm{Cl}, 61.1-93.1)$ were significantly improved from baseline $(54.2 ; 95 \% \mathrm{Cl}, 36.1-62.5 ; P<0.001)$. Scores on each subsection of the MoM survey also improved at 3 months, and median 1-year composite scores were significantly improved from baseline. (54.2 to 89 , $P=0.001$ ). Patient satisfaction with the education session was high, with a median satisfaction rating of 5 (a range of 4 to 5 ) on a 5-point satisfaction rating scale.
\end{abstract}

CONCLUSION: A community pharmacy-based menopause education program significantly increased scores on the MoM survey, and subjects were satisfied with this program.

KEYWORDS: Women's health, Menopause, Education, Community pharmacy, Management of Menopause survey, MoM survey

J Manag Care Pharm. 2004;10(5):442-48
I $t$ is estimated that 4,900 women enter menopause each day in the United States. ${ }^{1}$ Because menopause is associated with significant short-term symptoms and potential long-term consequences, the American College of Obstetricians and Gynecologists recommends that women be counseled annually about the treatment options for menopause. ${ }^{2}$ Despite this recommendation, there is evidence that woman are not receiving this information, and when they do, the sources of information or the information itself may be inadequate.

In a 1998 survey, only 38\% percent of women older than 50 years reported being counseled by their physician about hormone replacement therapy (HRT). ${ }^{3}$ A North American Menopause Society-Gallup telephone-based survey of 833 women aged from 45 to 60 years found that 36\% received a majority of their information about menopause from their physician and only $44 \%$ were very satisfied with the information. ${ }^{4}$ Twenty-seven percent of respondents indicated that magazines or journals provided the majority of their information on menopause. Of those women who received information from their physician, $84 \%$ reported that their physician discussed HRT, while less than $2 \%$ reported that their physician discussed lifestyle modifications or other medication options. ${ }^{4}$

The National Committee for Quality Assurance (NCQA), a nonprofit organization that provides accreditation of managed care organizations (MCOs) in the United States, recognized the need to address the lack of menopause counseling provided to women by health care providers. In 2000, NCQA incorporated the Management of Menopause (MoM) survey into the Health Plan Employer Data and Information Set (HEDIS) performance measures. HEDIS measures are used to assess the quality of care provided by MCOs and are an important component of the accreditation process. ${ }^{5}$

The MoM survey was designed to evaluate the provision of menopause counseling to members of MCOs. The survey assesses 3 aspects of menopause counseling and includes items pertaining to exposure, breadth, and personalization of counseling (sample items in Figure 1). MoM items in the breadth of counseling subsection focus on information related to the use of HRT and alternative approaches to managing the health consequences of menopause such as hot flashes, osteoporosis, Alzheimer's disease, and cardiovascular disease. At the time this HEDIS measure was developed in the late 1990s, evidence regarding the risks and benefits of HRT, particularly with respect to cardiovascular disease, was limited since the findings from the Women's Health Initiative (WHI) study were not yet available. ${ }^{6}$ 


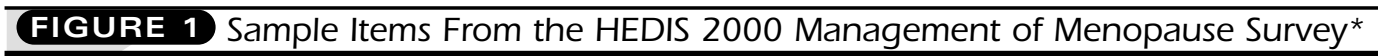

Items pertaining to exposure to counseling:

5. In the last 2 years, have you talked with a doctor, nurse or other health professional in your health plan about ways to deal with menopause?

- Yes Go to Question 7

- No Go to Question 6

7. In the last 2 years, were you given any information about ways to deal with menopause by your doctor's office or your health plan

_ Yes Go to Question 9

- No Go to Question 8

Items pertaining to breadth of counseling:

9. In the last 2 years, were you informed that hormone replacement therapy may help prevent heart disease?

- Yes

- No

- Can't recall

Items pertaining to personalization of counseling:

19. In the last 2 years, did the information you got about ways

to deal with menopause address your personal concerns?

- Yes

- No

- Can't recall

13. In the last 2 years, were you informed that there are ways other than taking hormones which help prevent heart disease?

- Yes

- No

- Can't recall

* A copy of the complete 22-item survey is available at the Office of Personnel Management

(available at: http://www.opm.gov/insure/02/html/standard/surveys/momsurvey_3u.html; accessed March 13, 2004).

Initial pilot testing of the MoM survey instrument provided further evidence of the need to provide additional menopause counseling. The survey yielded a mean overall composite score of 52 out of a possible 100.5 Similar results were demonstrated during the first year the instrument was administered nationally, in 1999; the mean composite score for the 149 MCOs reporting MoM survey data was 56.6. ${ }^{7}$ Little improvement was seen the following year, as the mean composite score reported in 2000 was 56.8. ${ }^{8}$ The low overall composite score coupled with the lack of change during the first 2 years of administration suggested there was room for improvement in the area of women's health education regarding the management of menopause.

Pharmacists have demonstrated their ability to provide patient education and monitoring services in the community pharmacy setting. ${ }^{9-11}$ In addition, mechanisms by which community pharmacists can document outcomes and obtain reimbursement for such cognitive services-outcomes-based pharmacist reimbursement (OBPR) — have been developed and evaluated. ${ }^{12}$ Community pharmacists have been identified as potentially playing a key role in improving the education of women regarding the management of menopause. ${ }^{13}$ By providing menopausal education in this setting, pharmacists can increase women's knowledge and understanding of this topic to help open the lines of communication with their health care providers.

A community pharmacy-based menopause education program could also assist MCOs in improving scores on the MoM survey and would be a seemingly ideal opportunity for OBPR for MCOs. A description of the process for developing and implementing a women's health education program in commu- nity pharmacy settings has been previously published. ${ }^{14}$ However, studies evaluating the impact and outcomes of such services are not available.

This project was designed to assess the outcomes of a community pharmacy-based menopause education program. Primary objectives included determining if the education program could improve MoM survey scores and increase the number of women who discuss treatment options with their physicians. Secondary objectives included assessing patient satisfaction with the education session and examining changes in diet, lifestyle, and medication use posteducation.

\section{Methods}

Seven community pharmacies ( 2 independent and 5 chain) were selected to participate in the project based on prescription volume, patient population demographics, and interest in the project. Pharmacists from these sites were selected to participate based on their overall relationship with their patients, patient communication skills, and interest in the project. None of the participating pharmacies or pharmacists specialized in the area of women's health education. All pharmacists were licensed to practice pharmacy in New York State. Study pharmacists participated in a 6-hour continuing education curriculum provided by the investigators that included information regarding short- and long-term consequences of menopause. In addition, pharmacologic and nonpharmacologic treatment options for the management of these consequences were reviewed along with study procedures. An emphasis was placed on (a) having the pharmacists determine the specific information needs of 
(FIGURE 2 Education Session Topic Checklist

Menopause Education Session

Before session, review the Jacobs Institute for Women's Health guidelines and the pocket guide.

1. Patient's existing knowledge and concerns

2. Overview of menopause:

Defined as absence of menstrual bleeding due to loss of ovarian function

- Short-term effects of estrogen deficiency

L Long-term effects of estrogen deficiency

3. Treatment options:

- General nonpharmacologic and preventive medicine: REDUCE RISK!

- Menstrual irregularity (can still conceive)

- Hot flashes

- Vaginal dryness

口 Osteoporosis

- Cardiovascular disease

- Colon cancer

4. Benefits and risks of treatment options:

- HRT benefits

口 HRT risks

口 Bisphosphonates benefits/risks

口 Raloxifene benefits/risks

- Statins (i.e., Lipitor, Mevacor, etc.)

5. Remaining questions and concerns:

Q Questions for their physician

- Plans to see physician to discuss

HRT = hormone replacement therapy; $C H D=$ coronary heart disease .

each subject and (b) enhancing their ability to answer questions frequently posed by women by discussing common areas of concern or confusion.

The study protocol was approved by the Investigational Review Board (IRB) of Albany Medical Center prior to initiating the project. Women aged 47 to 55 years who were pharmacy patrons and able to provide informed consent were eligible to participate. Subjects were enrolled and surveys were returned during a 2-year period from January 2001 to January 2003. Women unable to complete a self-administered survey and employees of the pilot pharmacies were not eligible.

IRB-approved study brochures were attached to the prescription bags of eligible women at pilot pharmacies, and signs posted in the pharmacy were used to recruit subjects. Women interested in participating were asked to schedule an appointment with the pharmacist for an education session. No monetary compensation was provided to subjects for participating, and informed consent was obtained from all subjects.

A baseline questionnaire, which contained the MoM survey, was distributed by study pharmacists or pharmacy students prior to the education session. The baseline questionnaire also included questions regarding menopausal status, estrogen replacement therapy (ERT) or HRT use, current medication use, and past medical and family history. The questionnaire was presented in the same format, font, and lettering as the original validated MoM survey, with no additional instructions for completion provided by study personnel.

A checklist, devised by the pharmacists during the continuing education program and enhanced by the investigators, was provided to guide pharmacists through the patient education session (Figure 2). Subjects received verbal and written education on various topics related to the management of menopause. Pharmacists were encouraged to tailor the patient education session to meet the subject's individual needs and were prompted by the checklist to identify suggested content for the education program. During the session, the short- and long-term consequences of menopause were reviewed. Both pharmacologic and nonpharmacologic prevention and treatment options were reviewed along with the known benefits and risks of those options. Pharmacists answered questions and referred women to their prescribers for further discussion and therapy decisions as needed. While the duration of each session was not limited to a specific length of time, most sessions lasted approximately 30 minutes. No medications were initiated or altered by the pharmacist during this study.

Subjects were contacted by mail at 3 months and 1 year posteducation to complete a follow-up survey with a self-addressed stamped envelope to be returned to the investigators. Four weeks after the initial mailing, a second mailing was sent to nonresponders. The questionnaire was the same as the baseline MoM survey instrument but included 4 additional questions to obtain information regarding secondary outcomes. This included questions assessing self-reported dietary, lifestyle, and medication modifications made during the study period; the number of subjects who initiated discussion with their health care provider; subject rating of the information provided by pharmacists; and satisfaction with the program.

\section{Outcomes}

The primary outcome of this study is a comparison of median MoM survey composite scores from baseline to 3 months and 1 year posteducation. Secondary outcomes include comparisons of median MoM survey subscores from baseline to 3 months and 1 year posteducation, satisfaction with the education session, and an assessment of self-reported changes in diet, lifestyle, and medication use 3 months and 1 year posteducation. The MoM survey is a self-administered, validated questionnaire, appropriate for an 8th-grade reading level, designed to assess the provision of menopause counseling during the past 2 years or at any time. ${ }^{7}$ The survey yields an overall composite score and 3 subscores derived from 16 questions. The subscores cover 
3 dimensions of menopause counseling including exposure to counseling (4 questions), breadth of counseling (9 questions), and personalization of counseling (3 questions). Subscores and the overall composite score are derived using a scoring rubric and formula provided by NCQA. The maximum score for each subcomponent and the overall composite score is 100 points. The composite score is obtained by taking the average of the 3 subscores. ${ }^{5}$ Permission was obtained from NCQA to use the MoM survey for this research project.

\section{Statistical Analysis}

Statistical analyses were performed using Minitab 13.20 and Statistix 7.0 software. Descriptive statistics were used to report survey results. In this study, MoM survey results are reported primarily as medians because the method of scoring this instrument results in data that are not continuous in nature. However, it should be noted that NCQA reports national MoM survey scores as means. ${ }^{5}$ Therefore, both means and medians are reported in MoM survey scores. A field test conducted by NCQA in 1998 using the MoM survey revealed a mean overall composite score of 52 and a range of 45 to 55 (standard deviation not specified). ${ }^{5}$ Assuming an average baseline composite score of 50, the goal of this study was to increase the MoM survey composite score by $10 \%$ after patient education. Assuming an alpha of 0.05 , beta of 0.2 , and a standard deviation of 5 points, at least 16 subjects would be required to complete the 3-month and 1 -year questionnaires..$^{15}$ Survey scores at 3 months and 1 year were compared with baseline scores using the Wilcoxon signed rank test. Differences in the rating of information provided by other health care professionals at baseline, 3 months, and 1 year were assessed using the Kruskal-Wallis test.

\section{Results}

A total of 31 subjects enrolled in and completed the education session and a baseline MoM survey during the 2-year study enrollment period from January 2001 to January 2003. However, the majority of subjects were enrolled during 2001 and early 2002. Subject characteristics are listed in Table 1. Only 1 subject did not keep a scheduled appointment. The majority of subjects were either perimenopausal or postmenopausal and had never used ERT/HRT. Few subjects reported a history of osteoporosis or coronary artery disease. Approximately one quarter of the subjects reported a family history of breast cancer.

Table 2 shows both median and mean results on the MoM survey. Twenty-four and 16 subjects completed and returned 3-month and 1-year follow-up surveys, respectively. Among the subcomponents of the survey, scores for personalization of counseling were lowest at baseline. Scores for exposure to counseling were consistently highest at baseline, 3 months, and 1 year. Compared with baseline, median 3-month scores were significantly increased for all components of the survey, including the composite score $(P<0.001)$. At 1 year, statistically significant

\section{TABLE 1 Subject Characteristics ( $N=31)$}

\begin{tabular}{lr}
\hline Age (mean \pm SD) & $51.4 \pm 2.4$ \\
Current menopausal status, number (\%) & $5(16)$ \\
Have not reached menopause & $16(52)$ \\
Going through menopause & $6(19)$ \\
Already gone through menopause & $4(13)$ \\
Don't know & \\
History of ERT/HRT use, number (\%) & $23(74)$ \\
Never used & $6(19)$ \\
Currently using & $2(7)$ \\
Used in the past & $2(7)$ \\
Personal history of osteoporosis, number (\%) & $1(3)$ \\
Personal history of CAD, number (\%) & $8(26)$ \\
Family history of breast cancer, ${ }^{*}$ number (\%)
\end{tabular}

* Defined as first-degree relative diagnosed with breast cancer. $S D=$ standard deviation; $E R T=$ estrogen replacement therapy; $H R T=$ hormone replacement therapy; $C A D=$ coronary artery disease.

TABLE $2 \longdiv { \text { Management of Menopause Survey Scores } }$

\begin{tabular}{|c|c|c|c|}
\hline Survey Component & $\begin{array}{l}\text { Baseline } \\
(\mathrm{N}=31)\end{array}$ & $\begin{array}{l}3 \text { Months* } \\
(\mathrm{N}=24)\end{array}$ & $\begin{array}{c}1 \text { Year } \dagger \\
(\mathrm{N}=16)\end{array}$ \\
\hline $\begin{array}{l}\text { Exposure to counseling } \\
\text { Median }(95 \% \mathrm{CI}) \ddagger \\
\text { Mean }( \pm \mathrm{SD})\end{array}$ & $\begin{array}{l}100(50-100) \\
67.2(46)\end{array}$ & $\begin{array}{l}100(83.4-100) \\
86.1(33.9)\end{array}$ & $\begin{array}{l}100(50-100) \\
81.3(40.3)\end{array}$ \\
\hline $\begin{array}{l}\text { Breadth of counseling } \\
\text { (95\% CI) } \\
\quad \text { Median }(95 \% \text { CI) } \\
\text { Mean }( \pm \text { SD) }\end{array}$ & $\begin{array}{l}58.3(37.5-62.5) \\
49.1(35.1)\end{array}$ & $\begin{array}{l}70.9(54.0-83.4) \\
68.7(34.3)\end{array}$ & $\begin{array}{l}100(50-100) \\
79.2(35.8)\end{array}$ \\
\hline $\begin{array}{l}\text { Personalization of } \\
\text { counseling } \\
\text { Median }(95 \% \mathrm{CI}) \\
\text { Mean }( \pm \text { SD) }\end{array}$ & $\begin{array}{l}33.3(16.7-50) \\
32.3(30.5)\end{array}$ & $\begin{array}{l}100(50-100) \\
70.8(38.5)\end{array}$ & $\begin{array}{l}100(50-100) \\
75(35.5)\end{array}$ \\
\hline $\begin{array}{l}\text { Composite score } \\
\text { Median }(95 \% \mathrm{CI}) \\
\text { Mean }( \pm \mathrm{SD})\end{array}$ & $\begin{array}{l}54.2(36.1-62.5) \\
49.5(32.1)\end{array}$ & $\begin{array}{l}86.1(61.1-93.1) \\
75.2(31.1)\end{array}$ & $\begin{array}{l}89(50-94.5) \\
78.5(32.6)\end{array}$ \\
\hline
\end{tabular}

* $P<0.001$ for comparison of baseline to 3-month median scores for all components. $\dagger P=0.002,0.001$, and 0.001 for comparison of baseline with 1-year for breadth, personalization, and composite median scores, respectively. Comparison for exposure to counseling was not statistically significant at 1 year $(P=0.06)$.

\# Confidence intervals for the Wilcoxon test that was used to compare the baseline values with 3 months and 1 year are based upon the rank order of the data but are expressed as the data value associated with the rank. Because the distribution of our data was such that most scores were 100, the maximum value, the data values for both the median, and the upper $95 \%$ confidence limit had the same value.

increases were seen for all components except exposure to counseling.

Table 3 lists results of the baseline and follow-up survey questions regarding satisfaction with the education provided by health care providers and study pharmacists. Median satisfaction scores for information provided by other health care providers were significantly elevated from baseline at 3 months $(P=0.01)$ and 1 year $(P=0.02)$. Subjects were very satisfied 


\begin{tabular}{l|c|c|c}
\hline \multicolumn{3}{c}{ TABLE 3 Ouestionnaire Results at Baseline, } \\
3-Month, and 1-Year Follow-up
\end{tabular}

* The number of respondents to individual questions may be lower due to surveys containing omissions.

+ Rated on a scale of 0 to 10, $(0=$ worst possible information; $10=$ best possible information).

\# Rated on a scale of 1 to 5, ( 1 = not satisfied; 5 = highly satisfied).

$\S$ Percentages were calculated based on the number of subjects responding to this question.

$\| P=0.01$ for comparison of baseline and 3-month rating of information scores. II $P=0.02$ for comparison of baseline and 1-year rating of information scores. N/A = not applicable.

\section{TABLE 4 Impact of Education Session on Diet/Lifestyle Modifications}

Question:

Did you or your doctor make any lifestyle or medication changes as a result of the session?

\begin{tabular}{l|c|c}
\hline & $\begin{array}{c}3 \text { Months } \\
(\mathbf{N}=\mathbf{2 4})^{*}\end{array}$ & $\begin{array}{c}\mathbf{1} \text { Year } \\
(\mathbf{N}=\mathbf{1 6})^{*}\end{array}$ \\
\hline Number responding "Yes" (\%) $\uparrow:$ & & \\
$\quad$ Diet & $4(20)$ & $6(40)$ \\
Exercise & $4(20)$ & $5(31)$ \\
Prescription medications & $2(10)$ & $2(13)$ \\
Vitamins or minerals $\neq$ & $7(35)$ & $2(13)$ \\
\hline
\end{tabular}

* The number of respondents to individual questions may be lower due to surveys containing omissions.

$\dagger$ Percentages were calculated based on the number of subjects responding to this question.

\# Eight subjects reporting specifically starting calcium either at 3 months or 1 year posteducation.

with the pharmacist education sessions, and median rating scores for the information provided by pharmacists was 9.0 and 8.0 at 3 months and 1 year, respectively (scale 0 to 10 , with $0=$ worst possible information, $10=$ best possible information).
The large majority of subjects reported that the education session improved their knowledge of the topic; however, only about half of the subjects reported discussing the education session with their physicians.

Changes in diet, lifestyle, and medication use among study subjects at 3 months and 1 year are listed in Table 4. Changes in diet and vitamin/mineral supplement use (primarily calcium supplementation) were reported in a relatively small number of subjects at 3 months. At 1 year, 50\% of responding subjects reported changes in vitamin/mineral supplement use. Few subjects reported changes in prescription medication use at 3 months or 1 year.

\section{Discussion}

Overall, the MoM survey results in this study were consistent with national means reported by NCQA during a similar time period. In 1999 and 2000, mean composite scores of 56.7 and 56.9 were reported, respectively. ${ }^{7.8}$ In this study, a comparable mean baseline composite score of 49.5 was found. Baseline scores for the subcomponents were also found to be similar. National means for exposure to counseling were 72.6 and 73.2 in 1999 and 2000, respectively. Breadth of counseling scores were 50.3 and 50.2 in 1999 and 2000, and personalization of counseling scores were 47.0 and 47.3 in 1999 and 2000.7.8 These scores are also comparable to the baseline scores for each component reported in this study (Table 2). As mentioned, the majority of subjects were enrolled during 2001 and early 2002. National mean scores for 2001, which would have been made available in 2002, were not reported by NCQA. However, the lack of substantial change in national mean scores from 1999 to 2000 provides evidence that the increase in scores seen in this study were not likely the result of systematic improvements that occur during the first few years of administration of a new measure.

Composite MoM survey scores were significantly increased from baseline at 3 months and 1 year posteducation. Approximately one half of the subjects reported discussing menopause with their physician following the education session. Considering the low composite MoM scores seen at baseline, it is likely that the education session was responsible for prompting women to discuss menopause-related issues. Baseline scores for "personalization of counseling" were found to be the lowest among the survey's 3 subcomponents, consistent with the findings from national administration of the survey in 1999. ${ }^{7}$ This suggests that information currently provided to women is often not patient-specific. A study by Clinkingbeard et al. found that almost one half of the 665 women surveyed reported leaving medical appointments with unanswered questions regarding menopause. ${ }^{16}$ Time constraints on physicians may be a contributing factor to the lack of personalized information provided to women on this topic. The training-education session provided by pharmacists in this study was specifically designed to address the personal 
educational needs of each study participant.

Our findings of low composite baseline scores confirm previous data suggesting the lack of information regarding menopause provided to women., ${ }^{3,416}$ The majority of subjects in our study were either premenopausal or perimenopausal. An observational study by Gallagher et al. examined the relationship between women's health characteristics and receipt of counseling regarding HRT by their health care provider. Only $40 \%$ of perimenopausal women and $13 \%$ of premenopausal women reported receiving counseling. In contrast, $79 \%$ of postmenopausal women reported receiving counseling. ${ }^{17}$ Given the importance of individualizing the decision to initiate HRT and the need for involving women in the decision-making process, it would likely benefit women to obtain counseling early during the years immediately premenopause or perimenopause. Gallagher et al. also found that only $27 \%$ of women who had not previously taken HRT received counseling. ${ }^{17}$ This further underscores the need for counseling prior to initiating therapy for menopause management as was the case for most women in this study (74\% had never received HRT/ERT).

Changes in the MoM survey composite score from baseline to 3 months and 1 year in this study represented improvement of approximately $60 \%$. This increase should be of interest to MCO decision makers. One of the underlying goals of this study was to determine the ability of pharmacists to improve performance scores of a HEDIS 2000 measure, potentially producing evidence to support the reimbursement for a pharmacy service that would be valuable to MCOs. Further incentive to this end was provided by a large survey of MCO customers performed in 1999 that found a positive relationship between the receipt of menopausal counseling and reenrollment rates with the MCO. ${ }^{18}$

By bridging the gap of information that currently exists between patients and physicians in this area, and increasing exposure to menopause counseling, pharmacists could have an impact on the satisfaction of MCO customers while improving patient care. Reimbursing pharmacists to provide this type of service to large numbers of patients could benefit MCOs in a number of ways. This intervention could be used to improve reenrollment rates and customer satisfaction as well as assist in the accreditation process by improving scores on an NCQAHEDIS measure. Evaluating the potential economic benefits and associated costs of providing this service was beyond the scope of this study, but our findings provide support for further investigation of the benefit-to-cost ratio of pharmacist-provided menopausal education of MCO members.

This study found relatively little impact of education on self-reported diet, lifestyle, and medication use. A similar study by Hunter and O'Dea found no changes in health behaviors in 86 premenopausal and perimenopausal women followed for 5 years after a health education intervention. ${ }^{19}$ Half of the subjects in the present study had reported changes in vitamin and mineral use at 1 year, primarily initiation of calcium supplementation.

\section{Limitations}

An inherent limitation of the MoM survey is the potential for recall bias because subjects are asked about information provided to them in the past 2 years. Also, there is a likely self-selection bias since this study involved only women who volunteered to receive menopause education from pharmacists.

This study did not have a control group, and it is therefore possible that the increase in composite MoM survey scores observed in this study was due to several factors, one of which was the education sessions provided by pharmacists. Results from the WHI study were published in July 2002. The media attention surrounding this study may have increased the percentage of women discussing this topic with their physician. ${ }^{6}$ In addition, the WHI study results may have stimulated MCOs to increase the amount of information provided to patients regarding this topic. However, 27 out of the 31 subjects in this study were enrolled prior to July 2002. In addition, among the 24 3-month surveys completed, 19 were returned prior to this date. Based on this time frame of subject enrollment as well as the fact that the 1-year scores in this study were similar to those at 3 months (Table 2), it is unlikely that the WHI study had a significant impact on the results of this study.

Findings from the WHI, most notably the lack of beneficial effects of HRT on cardiovascular outcomes and the increased risk of breast cancer, caused NCQA to suspend use of the MoM survey in 2004-various items in the survey (Figure 1) related to the effects of HRT on cardiovascular disease were deemed inappropriate given the findings of the WHI study. As a result, NCQA was examining the content of the MoM survey instrument at the time that this article was prepared and was considering ways to revise the instrument to reflect changes in practice or use an alternative method to capture information regarding rates of menopause counseling. ${ }^{7}$ While the content and method of use of the MoM survey are uncertain at the time of this article, the need for patient and MCO member education regarding menopause is more important than ever in the aftermath of the considerable publicity that surrounded the release of the initial WHI findings in mid-2002 and the findings released thereafter.

The enrollment process of this study may have contributed to the inclusion of women who were already actively seeking information related to menopause. These patients may have sought additional menopause information from their other health care providers or other sources regardless of participating in this study, which could have contributed to high MoM survey scores. However, the low baseline MoM survey composite scores indicate that our subjects had not sought or received this information in the recent past.

Finally, the method of determining changes in diet, lifestyle, 
and medication use was limited to patient self-reports that would require a control group to confirm the relationship of the study intervention to these changes. The sample size of this pilot study, while sufficient in terms of determining statistical differences in MoM survey scores, was small. A study using a control group and a larger sample size is necessary to confirm our findings.

\section{Conclusion}

A community pharmacy-based menopause education program increased the composite scores on the MoM survey from a median baseline value of 54.2 to 86.1 and 89.0 at 3 months and 1 year posteducation, respectively. These scores suggest that women who received pharmacist education were exposed to menopausal counseling of adequate breadth and personalization. Subjects were satisfied with the education provided by pharmacists, and the education session had a modest impact on self-reported dietary and lifestyle changes but not on prescription drug use. Given the favorable effects of this intervention on customer satisfaction and probable NCQA-HEDIS measures of health plan quality of care, MCOs should consider reimbursement for pharmacists providing this professional service.

\section{ACKNOWLEDGMENTS}

The authors would like to acknowledge Robert Hamilton, PharmD, professor, Albany College of Pharmacy, Albany, NY, for his assistance in the statistical analysis in this study, and Sarah Shih, MPH, senior health care analyst, National Committee for Quality Assurance, Washington, DC, for her assistance with obtaining NCQA MoM survey data.

\section{DISCLOSURES}

This project was presented as a poster (\#158) at the American College of Clinical Pharmacy Spring Practice and Research Forum in Palm Springs, California, April 29, 2003. Funding for this research was provided by Wyeth Pharmaceuticals, Inc., and was obtained by author Jennifer Cerulli. She has received honoraria from Wyeth for participating as a continuing education speaker; author Mario M. Zeolla discloses no potential bias or conflict of interest relating to this article. Zeolla served as principal author of the study. Study concept and design were contributed primarily by Cerulli. Analysis and interpretation of data and drafting of the manuscript and its critical revision were the work of both authors and statistical expertise was contributed by both. Administrative, technical, and/or material support was provided by Robert Hamilton, PharmD

\section{REFERENCES}

1. The North American Menopause Society. About menopause. Statistical facts and figures, United States. Available at: http://www.menopause.org/aboutmeno/ overview.html. Accessed August 20, 2004.
2. American College of Obstetricians and Gynecologists. ACOG technical bulletin. Health maintenance for perimenopausal women. Int J Gynaecol Obstet. 1995;51(2):171-81.

3. Collins KS, Schoen C, Joseph S, et al. Health concerns across a women's lifespan: the Commonwealth Fund 1998 Survey of Women's Health. Available at: http://www.cmwf.org/publist/publist2.asp?CategorylD=14. Accessed June 26, 2003.

4. Utian WH, Schiff I. NAMS—Gallup survey on women's knowledge, information sources, and attitudes to menopause and hormone replacement therapy. Menopause. 1994;1(1):39-48

5. The National Committee for Quality Assurance. HEDIS 2000. Vol. 3. Specifications for survey measures. Washington DC: NCQA; October 1999

6. Rossouw JE, Anderson GL, Prentice RL, et al. Writing Group for the Women's Health Initiative Investigators. Risks and benefits of estrogen plus progestin in healthy postmenopausal women: principal results from the Women's Health Initiative randomized controlled trial. JAMA. 2002;288(3):321-33.

7. Shih SC, Bost JE, Pawlson LG. Standardized health plan reporting in four areas of preventative health care. Am J Prev Med. 2003;24(4):293-300.

8. The National Committee for Quality Assurance. Management of menopause: informed choices. Washington DC: NCQA; 2001

9. Cranor CW, Bunting BA, Christensen DB. The Asheville Project: long-term clinical and economic outcome of a community pharmacy diabetes care program. J Am Pharm Assoc. 2003;43(2):173-84.

10. Warrick C, Wutoh AK, Corria-McDow Z, Emekalam A. Prostate cancer education in the Washington, DC, area. J Natl Med Assoc. 2002;94(11):963-70.

11. Tsuyuki RT, Johnson JA, Teo KK, et al. A randomized trial of the effect of community pharmacist intervention on cholesterol risk management: the Study of Cardiovascular Risk Intervention by Pharmacists (SCRIP). Arch Intern Med. 2002;162(10):1149-55.

12. Farris KB, Kumbera P, Halterman T, Fang G. Outcomes-based pharmacist reimbursement: reimbursing pharmacists for cognitive services. J Manag Care Pharm. 2002;8(5):383-93.

13. Cook R. Workshops: recommendations and plans of action for meeting the HEDIS 2000 management of menopause measure: pharmacy team recommendations. Am J Manag Care. 2000;6(suppl 14):774-77.

14. Knowlton CH, Thomas OV, Zarus SA, Buttaro ML. Women's health education: an opportunity for community pharmacy. J Am Pharm Assoc. 1997;37(1):109-22.

15. Stolley PD, Strom BL. Sample size calculations for clinical pharmacology studies. Clin Pharmacol Ther. 1986:489-90.

16. Clinkingbeard C, Minton BA, Davis J, McDermott K. Women's knowledge about menopause, hormone replacement therapy (HRT), and interactions with health care providers: an exploratory study. J Womens Health Gend Based Med. 1999;8(8):1097-1102.

17. Gallagher TC, Geling OC, Fitzgibbons J, Aforismo J, Comite F. Are women being counseled about estrogen replacement therapy? Med Care Res Rev. 2000; 57(suppl 2):72-92.

18. National Health Information LLC. Menopause management boosts patient satisfaction and re-enrollment rates. Dis Manage Advis. 2000;6(8):120-23.

19. Hunter M, O'Dea I. An evaluation of a health education intervention for mid-aged women: five-year follow-up of effects upon knowledge, impact of menopause and health. Patient Educ Couns. 1999;38:249-55. 